\title{
Performance Analysis of Packet Aggregation in WLANs with Simultaneous Multi-user Access (Work in Progress)
}

\author{
Andreas Könsgen, Md. Shahidul Islam, Andreas Timm-Giel, \\ and Carmelita Görg \\ Communication Networks \\ Center for Computing Technologies (TZI) \\ University of Bremen \\ Otto-Hahn-Allee 1, 28359 Bremen, Germany \\ ajk@comnets . uni-bremen.de
}

\begin{abstract}
A cross-layer wireless LAN system is considered which transmits packets for a number of users simultaneously using OFDMA or SDMA transmission. Due to varying packet lengths and physical bitrates for each user, this results in different transmission times so that users with faster transmissions have to wait until the transmission of the slower users is complete. Packet aggregation can reduce the loss of airtime and enhance the throughput without affecting the delay by filling in the gaps with additional packets which are ready for transmission. In this paper, the throughput is determined analytically and by simulation with and without packet aggregation for some given distribution functions of the packet size and the transmission time. For a more realistic channel model, the throughput enhancement due to packet aggregation is determined by simulations. It is shown that dependent on the distribution of the different flows, a significant throughput enhancement can be achieved.
\end{abstract}

Keywords: wireless LANs, multi-user access, packet aggregation.

\section{Introduction}

This paper reports about work in progress where a cross-layer based WLAN transmission system is designed which considers user quality-of-service demands from the application layer, queue filling states on the MAC layer and the radio channel conditions on the PHY layer. The general concept of the system was introduced in [1] and extended by QoS support in [2]. In each turn of the scheduling process, the MAC scheduler assigns a priority to the data packet at the top of each queue according to throughput and delay requirements of the application. An OFDMA/SDMA platform was introduced in [3] which provides simultaneous transmission to multiple users by adaptively allocating channel resources to each of them acording to the determined priorities. The decisions of the channel allocation algorithm is then fed back to the queues which have to keep track how much data was transferred for each user. 
The cross-layer transmission system is located in the access point which has full control of the channel access, similar to the Hybrid Coordination Function Controlled Channel Access (HCCA) specified in IEEE 802.11e. In this paper, only the downlink from the access point to the mobile stations is considered.

In the previous investigations, it was assumed that the packet sizes for all users were constant and equal. In this paper, the scenario is extended to variable packet size which, when the users face variable transmission rates because of fading effects of the radio channel, results in widely varying times that the individual users need to transmit a packet. The cross-layer scheduler adaptively allocates power to the different users and the OFDM subcarriers, which requires that the transmission of all users start at the same time. Hence they have to wait until the slowest user has completed his transmission before the next set of packets can be sent so that airtime is wasted unused.

This paper analyzes the gain which can be achieved if the above-mentioned unused airtime gaps are filled by aggregation of multiple packets. The basic idea is that each user usually has packets waiting in the queue which may fit into the gap. In the analysis discussed here, first the mean gap size which the users experience is calculated; after that, the upper boundary for the increase of the throughput is analytically determined. Finally, simulation results are given which illustrate the effect of packet aggregation.

The rest of the paper is organized as follows. An overview about related work is given in sect. 2. An analytical model for the packet aggregation is developed and elaborated in sect. 3 for simplified distribution functions which can be closely expressed. In sect. 4 the analytic calculations are compared with simulation results. Section 5 concludes the paper.

\section{Related Work}

Quality-of-service based scheduling in wireless LANs has become an important research topic in recent years. For example, in [4, a QoS scheduler is shown which is derived from an optimization problem and then simplified to a heuristic algorithm. QoS is also considered in the scheduler described in [5] where 802.11e transmission parameters are modified; 6] proposes a scheduler which takes a scheduling decision on a packet-level basis and considers the multiple PHY rates which a WLAN offers. In [7, demands by the application are considered by abstracting them to a single numerical value. An analysis of a channel state aware scheduler is given in [8] where the channel is modeled as a Markovian process. An enhanced channel model is used in [9] where the channel characteristics are included into a cross-layer approach for the scheduler. The algorithm discussed here is cross-layer based as well, considering a MIMO-OFDMA transmission; in this paper, the focus is on a theoretical analysis of the scheduler performance.

The introduced transmission system extends the proposal of the IEEE $802.11 \mathrm{n}$ standards draft, where centralized channel access is specified as Hybrid Coordinated Channel Access (HCCA). On the physical layer, MIMO is used, however only one user is served at a time using TDMA. The scheduler discussed here introduces the parallelized transmission using OFDMA or SDMA. 


\section{Calculation of the Capacity}

A scenario is considered where an access point simultaneously transmits packets to $n$ users. Each of the packets has a different transmission duration, which can result from varying channel conditions or packet lengths.

Each of the simultaneously transmitted packets take a different time for transmission. For all data flows which transmit their packet faster than the packet with the slowest transmission time, there is a time gap until the next transmission can be started. The algorithm which assigns the channel capacities for the data flows needs to know the priorities of the packets which are determined at the MAC layer so that a transmission start at the same time is required.

Because of the analytical calculation, simplified distribution functions for the packet size and the channel capacity are used for which closed expressions are available. For comparison, the distribution function for a more realistic simulated channel is shown afterwards along with the resulting throughput figures.

In the calculation, first the mean length of the packet with the longest transmission time interval and the mean lengths of the other (shorter) packets are calculated. From this, the mean length of the gap $\delta$ between the end of the transmission and the start of the next one is determined.

\subsection{Calculation of the Mean Gap Size}

The sample of the probability distribution function (PDF) for the transmission duration for flow $i$ at time $t$ is written as $f_{i}(t)$. The CDF is then

$$
F_{i}(t)=\int_{0}^{t} f_{i}(x) d x .
$$

The maximum transmission time within a turn of the scheduler is written as $x_{\max }=\max \left\{x_{1}, x_{2}, \ldots x_{n}\right\}$. The probability that $x_{\max }$ is shorter than a time $t$ is

$$
\begin{aligned}
P\left(x_{\max } \leq t\right) & =P\left(\max \left\{x_{1}, x_{2}, \ldots\right\} \leq t\right) \\
& =F_{1}(t) F_{2}(t) \ldots F_{n}(t) .
\end{aligned}
$$

Assuming the same distribution functions for all flows, there is $f_{i}(t)=f(t)$ and $F_{i}(t)=F(t)$ for all $i$, so that $P\left(x_{\max } \leq t\right)$ can be written as $(F(t))^{n}$.

The PDF for the transmission time of the longest packet $f_{\max }(t)$ is then

$$
\begin{aligned}
f_{\max }(t) & =\frac{\mathrm{d}}{\mathrm{d} t}(F(t))^{n} \\
& =n F(t)^{n-1} \cdot f(t) .
\end{aligned}
$$

To get the mean gap size $\delta$, the difference between the mean value of the longest packet and the mean value of an individual route is calculated:

$$
\delta=\int_{0}^{\infty} t \cdot f_{\max }(t) \mathrm{d} t-\int_{0}^{\infty} t f(t) \mathrm{d} t .
$$


In case of $n$ data flows with $f(t)$ being a uniform distribution, the minimum transmission time $T_{\min }$ and the maximum transmission time $T_{\max }$, one gets as a solution for (41):

$$
\delta=\frac{n T_{\max }+T_{\min }}{n+1}-\frac{T_{\max }+T_{\min }}{2} .
$$

For the exponential distribution with mean transmission time $\mu$, a close expression (4) as a function of the route number $n$ is not possible. When solving for a fixed number of $n=8$ which is used as the number of data flows in the example scenario which is discussed here, one gets as the solution

$$
\delta=\frac{481}{280} \mu \text {. }
$$

\subsection{Calculation of the Throughput}

After determining the time gaps, the throughput can be calculated. The calculations are done for uniform and exponential distribution of the transmission duration. When the time gap is filled for a certain data flow by packet aggregation and interruptions of the transmission due to interframe spacings and acknowledgement packets are neglected, a continuous transmission is possible. The available throughput is then equal to the physical bitrate. With the equations given for the mean gap size, the increase of the available data rate can then be calculated. In the following calculations, $S_{\max }$ is the throughput with packet aggregation, $S_{0}$ is the throughput without, $\Delta S=S_{\max }-S_{0}$ and $T_{\text {meanmax }}$ is the mean value of the TX time needed for the longest packet.

The delay of the packets is not increased by packet aggregation because only existing gaps are filled in by additional packets which otherwise would be transmitted later. No transmission is delayed in order to aggregate the packets.

The following calculations are presented for three cases, where either the packet size or the transmission rate or both are variable. For each of these cases, first the expression for a general distribution is given, after that the equations for uniform and negative exponential distributions are deduced.

Constant Transmission Rate, Variable Packet Size. When the TX rate $R$ is assumed to be constant and the packet size is subject to a general distribution, there is

$$
S_{0}=\frac{\text { MeanPacketSize }}{\text { MeanLongestTxTime }} .
$$

with

$$
T_{\text {meanmax }}=\int_{0}^{\infty} t \cdot f_{\max }(t) \mathrm{d} t .
$$

$f_{\max }$ can be calculated according to (11) to (4), where $x_{i}=P_{i} / R, P_{i}$ being the size of the packet of flow $i$ which is currently transmitted. 
Uniform distribution. For the increment of the throughput it can be calculated:

$$
\Delta S=R \cdot\left(\frac{n \cdot T_{\max }+T_{\min }}{n+1}-\frac{T_{\max }+T_{\min }}{2}\right) .
$$

Negative exponential distribution. There is no close expression which expresses $S$ as a function of $n$. In case of $n=8$ flows, there is

$$
\Delta S=\frac{481}{761} R .
$$

Constant Packet Size, Variable Transmission Rate. In this case, the transmission time is dependent on the physical transmission rate. Let $g(t)$ be the PDF of the transmission rate and $f(t)$ the PDF of the longest transmission time in a sample. In [10] it is shown that the PDF of the product $g(t) f(t)$ of dependent random variables $g(x)$ defined in interval $(c, d)$ and $f(x)$ defined in the interval $(a, b)$ is

$$
\mathrm{PDF}=\left\{\begin{array}{l}
\int_{a}^{\frac{\nu}{c}} g\left(\frac{\nu}{x}\right) f(x) \frac{1}{x} \mathrm{~d} x, a \cdot c<\nu<a \cdot d \\
\int_{\frac{\nu}{d}}^{b} g\left(\frac{\nu}{x}\right) f(x) \frac{1}{x} \mathrm{~d} x, a \cdot d<\nu<b \cdot d .
\end{array}\right.
$$

The average throughput is

$$
S_{\max }=\frac{\mathrm{E}[\nu]}{T_{\operatorname{meanmax}}}=\frac{\int_{0}^{\infty} \nu p(\nu) \mathrm{d} \nu}{T_{\operatorname{meanmax}}} .
$$

Uniform distribution. The PDF of the transmission rate is

$$
g(t)=\frac{1}{t^{2}\left(T_{\max }-T_{\min }\right)} .
$$

The PDF of the longest transmission time in a sample is

$$
f(t)=n \frac{\left(t-T_{\min }\right)^{n-1}}{\left(T_{\max }-T_{\min }\right)^{n}} .
$$

The PDF of the product $g(t) f(t)$ of the above random variables is

$$
p(\nu)=\left\{\begin{array}{l}
\frac{\left(v T_{\max }-T_{\min }\right)^{n}\left(T_{\min }+n T_{\max } \nu\right)}{\nu^{2}(n+1)\left(T_{\max }-T_{\min }\right)^{n+1}}, \frac{T_{\min }}{T_{\max }}<\nu<1, \\
\frac{T_{\min }+n T_{\max }}{(n+1)\left(T_{\max }-T_{\min }\right) \nu^{2}} \quad 1<\nu<\frac{T_{\max }}{T_{\min }} \\
-\frac{T_{\min }^{n+1}(\nu-1)^{n}(n \nu+1)}{(n+1)\left(T_{\max }-T_{\min }\right)^{n+1} \nu^{2}},
\end{array}\right.
$$


The throughput $S_{\max }$ with packet aggregation is then

$$
S_{\max }=\frac{\mathrm{E}(\nu)}{T_{\operatorname{meanmax}}}=\frac{\int_{1}^{\frac{T_{\max }}{T_{\min }}} \nu p(\nu) \mathrm{d} \nu+\int_{\frac{T_{\min }}{T_{\max }}}^{1} \nu p(\nu) \mathrm{d} \nu}{T_{\operatorname{meanmax}}}
$$

which can be solved analytically, however the expression is large and not shown here due to space reasons.

Without packet aggregation, the throughput is calculated as

$$
S_{0}=\text { PacketSize } / T_{\text {meanmax }},
$$

where $T_{\text {meanmax }}$ can be calculated according to the left fraction of expression (5).

Exponential distribution. In this case, the PDF of the transmission time can be written as

$$
p(t)=\lambda e^{-\lambda(t-s)}, s<t<m,
$$

where $s$ is the min. and $m$ the max. transmission time.

The PDF of the transmission rate is then

$$
g(t)=\frac{\lambda e^{\lambda\left(s-\frac{1}{t}\right)}}{t^{2}}, \frac{1}{m}<t<\frac{1}{s} .
$$

The PDF of the largest element in a sample is

$$
f_{\max }(t)=n\left(1-e^{-\lambda(t-s)}\right)^{n-1} \lambda e^{-\lambda(t-s)} .
$$

The PDF of the product between the largest element in a sample and the transmission rate for $n=8$ is

$$
h(\nu)=\left\{\begin{array}{l}
\frac{8 \lambda^{2}}{\nu^{2}} \int_{s}^{\nu \cdot m} x\left(1-e^{-\lambda(x-s)}\right)^{7} e^{\lambda\left(2 s-x-\frac{x}{v}\right)} \mathrm{d} x \\
\frac{8 \lambda^{2}}{\nu^{2}} \int_{\nu \cdot s}^{m} x\left(1-e^{-\lambda(x-s)}\right)^{7} e^{\lambda\left(2 s-x-\frac{x}{v}\right)} \mathrm{d} x
\end{array}\right.
$$

for $\frac{s}{m}<\nu<1$ and $1<\nu<\frac{m}{s}$, respectively.

The average throughput with packet aggregation is

$$
S_{\max }=\frac{\mathrm{E}[\nu]}{T_{\operatorname{meanmax}}}=\frac{\int_{\frac{s}{m}}^{l} \nu h(\nu) \mathrm{d} \nu+\int_{\frac{m}{s}}^{l} \nu h(\nu) \mathrm{d} \nu}{T_{\operatorname{meanmax}}} .
$$

The average throughput without packet aggregation $S_{0}$ can be calculated according to (7) where

$$
T_{\text {meanmax }}=\frac{761}{280 \cdot \lambda}+s
$$


Transmission Rate and Transmission Time Variable and Independent. Finally, it is assumed that the transmission rate and time behave according to the same distribution function, but both parameters are independent. This means that

$$
\text { MeanPacketSize }=\text { MeanTxRate } \cdot \text { MeanTxTime } .
$$

The throughput with packet aggregation $S_{\max }$ is equal to the mean transmission rate independent of the transmission rate distribution.

Uniform distribution. With $T_{\min }$ and $T_{\max }$ as described earlier and $R_{\min }, R_{\max }$ being the minimum and maximum rate, the mean throughput is

$$
S_{0}=\frac{(n+1)\left(R_{\min }+R_{\max }\right)\left(T_{\min }+T_{\max }\right)}{4\left(n T_{\max }+T_{\min }\right)}
$$

and

$$
\Delta S=\frac{R_{\min }+R_{\max }}{2} \cdot\left(\frac{n \cdot T_{\max }+T_{\min }}{n+1}-\frac{T_{\max }+T_{\min }}{2}\right) .
$$

Exponential distribution. The throughput without packet aggregation is

$$
S_{0}=\frac{280}{761} \text { MeanTxRate. }
$$

With $S_{\max }=$ MeanTxRate, the increment of the throughput $\Delta S$ can then easily be calculated.

\section{Simulation Results}

\subsection{Comparison with the Analytical Calculations}

The theoretical deductions are compared with simulations which model the scenario of an access point serving $n=8$ stations as it already has been described at the beginning of sect. 3. The simulator implements the generation and transmission of packets with given distributions of packet size and transmission time. The measurement values such as gap size and throughput are obtained by statistical evaluation.

First, the mean gap is determined by measuring the length of the longest packet and comparing it with the length of packets which are transmitted faster. Fig. 1 below shows the analytic results (given by the lines) and the simulation results (given by the circles). The relationship between the maximum resp. mean transmission time and the mean gap size is linear for both distribution functions. The analytic and the simulation results match with negligible difference.

Figure 2 shows the graphs for constant packet size and uniform resp. exponential distribution of the transmission time. The transmission rate is in this case dependent on the previously mentioned parameters. This scenario approximates the case that all users run applications such as file downloads or CBR video 

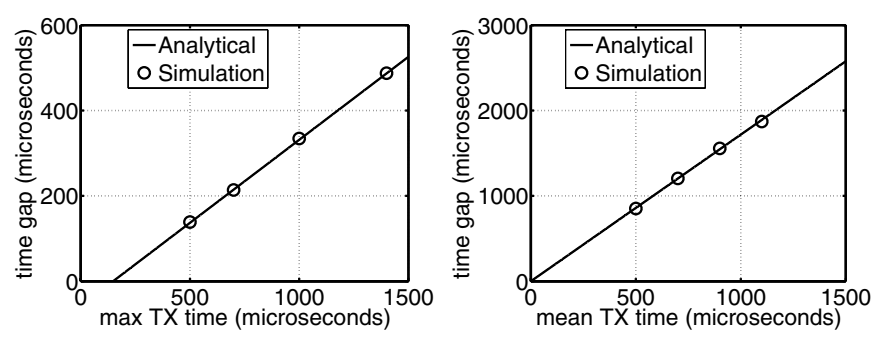

Fig. 1. Gap size for uniform and exponential distribution

transmissions where the packet size does not change. The achievable throughput is reduced when the transmission time is increased. The shape of the graph is similar for uniform and exponential distributions; they differ in the absolute values yielded for a particular packet size. The enhancement ratio of the throughput is increased the longer the transmission time becomes, which means it is in particular effective in case of bad channel conditions.

In Fig. 3, the graphs for constant transmission rate and uniform/exponential distributions of the transmission time are shown. In this case, the packet size is a dependent variable. This scenario would appear in reality if the users run different types of applications with variable packet size, but all have similar channel conditions. The graphs for the distributions without packet aggregation have different shapes: for the uniform distribution, it is reciprocal; for the negative exponential distribution, it is constant. Since the TX rate is assumed to be constant in this case, this means that the amount of enhancement increases with growing packet sizes. For the exponential distribution, the analytical results are slightly lower than the simulated ones. Due to the numeric processing, large values for the transmission time are not considered so that the numerically calculated packet length is slightly shorter then the analytical one which results in a higher throughput. Figure 4 shows the graphs for independent distribution functions for the transmission rate and the transmission time. In practice, this is the general case where users run different types of applications and face different channel conditions. The shapes of the result graphs are similar to the previous
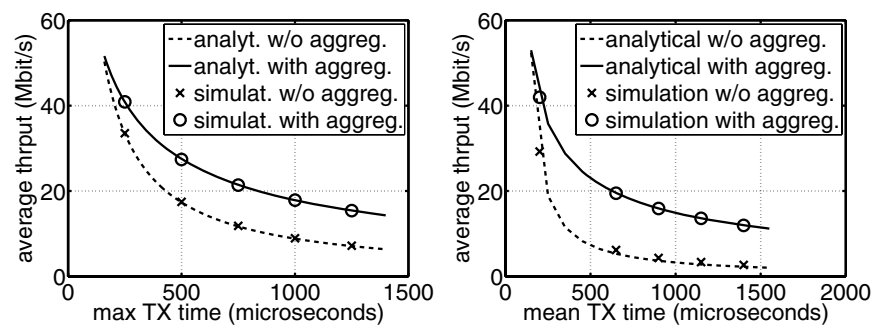

Fig. 2. Throughput for constant packet size and uniform/exp. distribution for TX time 

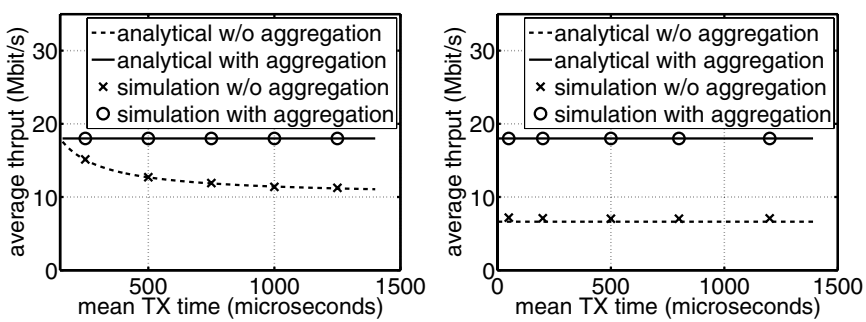

Fig. 3. Throughput for constant TX rate and uniform/exp. distribution of TX time
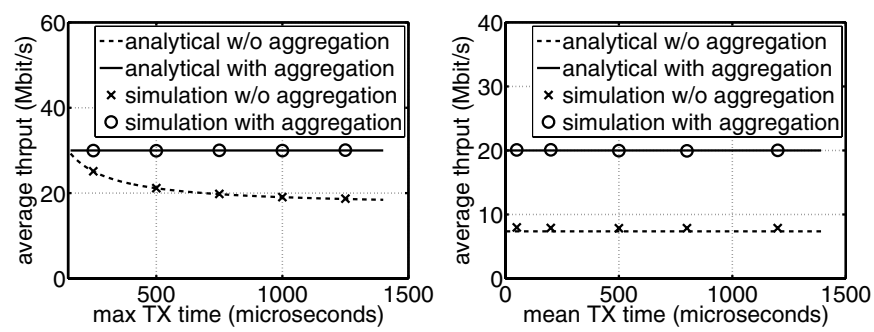

Fig. 4. Throughput for independent uniform/exp. distribution of TX rate and TX time

case where the transmission rate was constant; the same applies for the amount of enhancement due to packet aggregation. For the exponential distribution, the numerically calculated values are slightly higher than the analytical values due to same reason as in Fig. 3

\subsection{Realistic Channel Model}

For comparison, a scenario with a realistic channel model is also considered. Like in the previous analytical and simulated scenarios, an access point serves $n=$ 8 mobile stations using packet aggregation where each station receives one data flow. The load generator provides CBR distributed traffic for the flows 1, 2 and 4 and Poisson traffic for the other flows; the traffic load is configured individually for each flow. The packets for each flow are stored in the respective queue until they are served by the MAC scheduler. The flows 1 to 3 are time-critical due to a delay constraint; the scheduler tries to send a packet within max. $15 \mathrm{~ms}$ after arrival in the queue. The other flows are not delay-constrained, they are controlled according to the target throughput which corresponds to the offered load.

The channel capacities are determined based on the IEEE 802.11 TGn radio channel model proposed in [3] which is deployed here to implement a MIMO transmission with $M=2$ transmit antennas at the base station and $N=2$ receive antennas at each of the mobile stations, $K=8$ users and $L=52$ subcarriers. The model considers an indoor environment with moving obstacles which scatter the radio signal while it propagates from the sender to the receiver and result in varying 


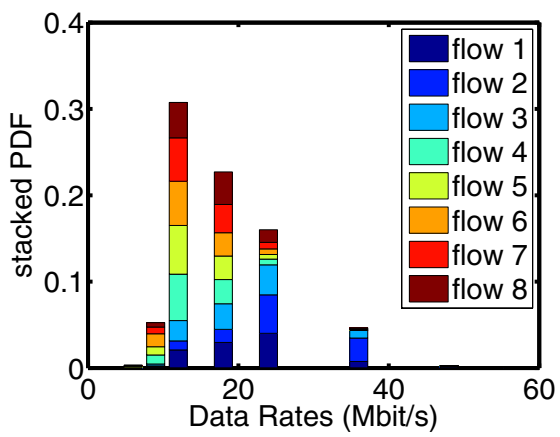

Fig. 5. Stacked PDF of the channel capacities for the different flows in case of realistic channel

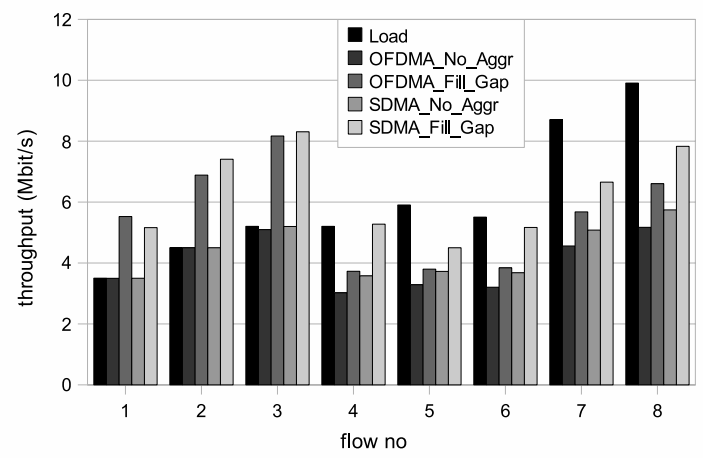

Fig. 6. Thrp. for the individual flows in case of realistic channel, constant packet size

channel conditions. An OFDMA or SDMA transmission is considered where each subcarrier is allocated to a particular user or subcarriers are shared between users based on spatial diversity. An important difference between the idealised scenarios discussed above and the realisitic channel model is that users in avarage may see different channel conditions regarding the mean capacity.

In Fig. 5] 6] and 7) measurements for a real channel are shown as it was described in the previous paragraph. The available data rates can be selected as in IEEE 802.11a or g systems between 6 and $54 \mathrm{Mbit} / \mathrm{s}$. Fig. 5 shows the stacked distribution function for the relative amount of packets which was transmitted at a certain data rate. The length of each bar shows the total probability that a certain data rate was used, the lengths of the sections inside each bar show the probability for a certain flow. The graph shows the behavior of the QoS-aware scheduler: the time-critical flows 1 to 3 are assigned more often to higher bitrates than the non-time-critical flows.

Figures 6 and 7 show the per-flow throughput for data flows with constant and variable packet size. Each figure shows results which were achieved by using 


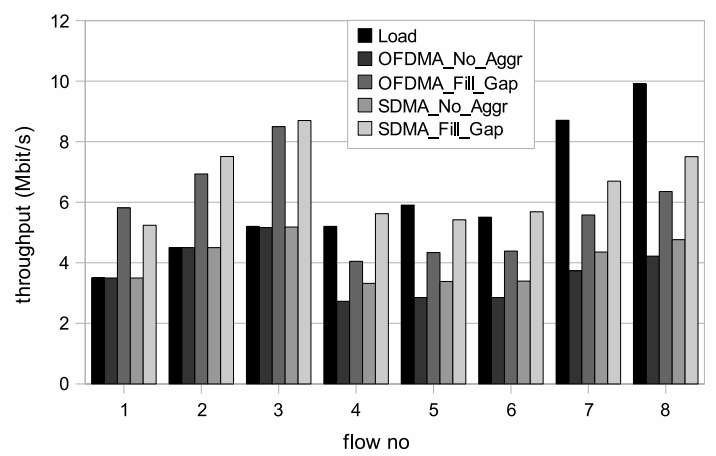

Fig. 7. Thrp. for the individual flows in case of realistic channel, variable packet size

OFDMA and SDMA as the method for the parallel transmission of packets. For each flow, the left bar denotes the offered load, the bars OFDMA_No_Aggr and SDMA_No_Aggr denote the throughput in case that no packet aggregation is used, respectively for OFDMA and SDMA. The bars OFDMA_Fill_Gap and SDMA_Fill_Gap show a hypothetical case, namely the throughput which could be achieved if the remaining gaps for each flow would be filled entirely with data, regardless of the offered traffic load. For the time-critical flows, this throughput is higher than the load, because for these flows the queues are usually empty due to the quick serivce of the packets. Therefore the gaps between the end of a packet transmission and the start of the next transmission are not filled in case of normal operation. Filling the gaps hence means that additional data beyond the offered load is transferred.

The flows 1 to 3 are real-time flows where the throughput must be kept. The remaining capacity is distributed among the other flows. The figures show that the time-critical flows are always served according to their needs. The throughput of the non-time-critical flows is increased in all scenarios when packet aggregation is used.

\section{Conclusion and Outlook}

A theoretical deduction was given how packet aggregation can enhance the throughput of a cross-layer scheduler with parallel transmissions. The achievable throughput increase was determined for different scenarios where the distribution of the packet size and the transmission time are either constant or according to a given distribution function. It was shown by analytical calculations and validated by simulations that packet aggregation significantly enhances the throughput. These results were compared with simulations based on a realistic channel model and with mixed time-critical and non-time-critical traffic which also showed a throughput improvement.

It was assumed that each flow fills the gap of air time completely to determine the theoretical upper boundary of the enhancement by packet aggregation. In 
practice, the packets to be aggregated have given sizes so that the gap might not be fully closed. Furthermore, it was assumed that each user always has data to be transmitted. In practice, a user might have a relatively low data rate so that there is no transmission requirement even if resources are available. Hence there is the need for more realistic simulations where the filling state of the queues for the different users are considered.

\section{References}

1. Könsgen, A., Herdt, W., Timm-Giel, A., Görg, C.: A Crosslayer Two-Stage Scheduler for Wireless LANs. In: Mobile and Wireless Communications Summit, Budapest, Hungary (2007)

2. Könsgen, A., Herdt, W., Timm-Giel, A., Wang, H., Görg, C.: An Enhanced Crosslayer Two-Stage Scheduler for Wireless LANs. In: Int. Symposium on Personal and Indoor Wireless Comm. (PIMRC), Athens, Greece (2007)

3. Kermoal, J.P., Schumacher, L., Pedersen, K.I., Mogensen, P.E., Frederiksen, F.: A Stochastic MIMO Radio Channel Model with Experimental Validation. IEEE Journal on Selected Areas in Communications. Work supported by IST project I-METRA IST-2000-30148 20(6) (2002)

4. Senst, A., Schulz-Ritticg, P., Croonen, D., Ascheid, G., Meyr, H.: A Wireless Revenue Based Scheduler with QoS Support. In: 41st Annual Allerton Conf. on Communications, Control and Computing, Monticello, IL, USA (2003)

5. Inan, I., Keceli, F., Ayanoglu, E.: An Adaptive Multimedia QoS Scheduler for 802.11e Wireless LANs. In: Proc. IEEE Int. Conf. on Communication, Istanbul, Turkey (2006)

6. Yuan, Y., Gu, D., Arbaugh, W., Zhang, J.: Achieving Packet-Level Quality of Service Through Scheduling in Multirate WLANs. In: Proc. Vehicular Technology Conference (VTC), Los Angeles, USA (Fall 2004)

7. Khan, S., Suhovniko, S., Steinbach, E.: Application-driven Cross-layer Optimization for Mobile Multimedia Communication using a Common Application Layer Quality Metric. In: Proc. 2nd Int. Symposium on Multimedia over Wireless (ISMW), Vancouver, CDN (2006)

8. Rom, R., Tam, H.P.: Analysis of Performance Trade-offs for an Adaptive Channelaware Wireless Scheduler. Springer Wireless Network Journal (December 2007)

9. Liu, Q., Zhou, S., Giannakis, G.B.: Queuing with Adaptive Modulation and Coding Over Wireless Links: Cross-Layer Analysis and Design. IEEE Transactions on Wireless Comm. 4(3) (May 2005)

10. Glenn, A.G., Leemis, L.M., Drew, J.: Computing the distribution of the product of two continuous random variables. Computational Statistics and Data Analysis 44(3) (January 2004) 